\title{
Percolation quantum phase transitions in diluted magnets
}

\author{
Thomas Vojta ${ }^{1}$ and Jörg Schmalian ${ }^{2}$ \\ ${ }^{1}$ Department of Physics, University of Missouri-Rolla, Rolla, MO 65409 \\ ${ }^{2}$ Department of Physics and Astronomy and Ames Laboratory, Iowa State University, Ames, IA 50011
}

(Dated: November 17, 2018)

\begin{abstract}
We show that the interplay of geometric criticality and quantum fluctuations leads to a novel universality class for the percolation quantum phase transition in diluted magnets. All critical exponents involving dynamical correlations are different from the classical percolation values, but in two dimensions they can nonetheless be determined exactly. We develop a complete scaling theory of this transition, and we relate it to recent experiments in $\mathrm{La}_{2} \mathrm{Cu}_{1-p}(\mathrm{Zn}, \mathrm{Mg})_{p} \mathrm{O}_{4}$. Our results are also relevant for disordered interacting boson systems.
\end{abstract}

Geometric criticality and quantum criticality are two distinct phenomena leading to universal scale invariant correlations. Both get combined in randomly diluted quantum magnets. Site or bond dilution defines a percolation problem for the lattice with a geometric phase transition at the percolation threshold [1]. Quantum fluctuations of the spins coexist with geometric fluctuations due to percolation. In this Letter, we address the question of whether or not these quantum fluctuations fundamentally change the percolation phase transition.

The generic phase diagram of a diluted magnet as function of impurity concentration $p$, quantum fluctuations strength $g$, and temperature $T$ is shown in Fig. 1 In the absence of both thermal and quantum fluctuations ( $g=T=0)$, magnetic long-range order survives for all concentrations $p \leq p_{c}$, where $p_{c}$ is the classical (geometric) percolation threshold. If $p>p_{c}$, no long-range order is possible as the system is decomposed into independent clusters. For $p<p_{c}$, magnetic order survives up to a nonzero temperature $T_{c}(p)$; but at $p_{c}$, it is destroyed immediately by thermal fluctuations, $T_{c}\left(p_{c}\right)=0[3,[4,[5,6]$.

Zero temperature quantum fluctuations are less effective in destroying magnetic order. Even at $p_{c}$, the perco-

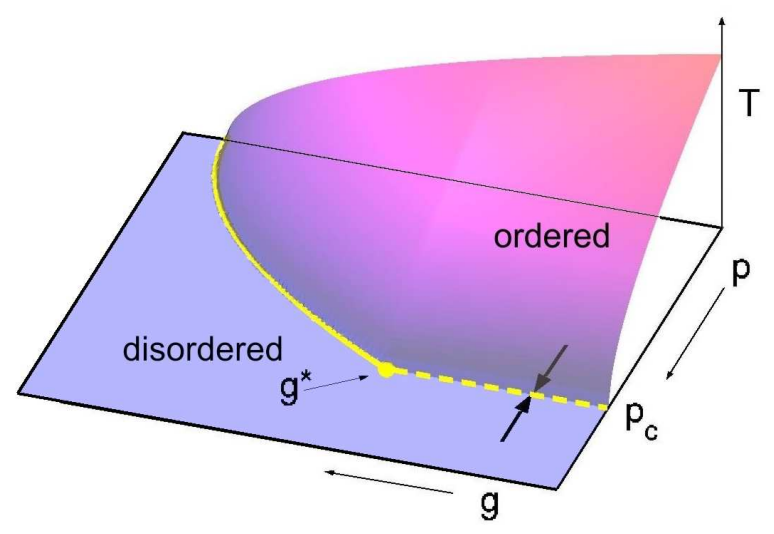

FIG. 1: Schematic phase diagram of a diluted magnet with impurity concentration $p$, temperature $T$, and quantum fluctuation strength $g$ [2]. There is a quantum multicritical point (big dot) at $\left(p_{c}, g^{*}\right)$. The zero temperature quantum phase transition across the dashed line is the topic of this Letter. lating cluster can remain ordered up to a nonzero fluctuation strength. This was found in the diluted transverse field Ising model [7, 8] where a multi-critical point with transverse field strength $g^{*}$ and $p=p_{c}$ emerges. At this point, both quantum and geometric fluctuations diverge. In $O(N)$ systems $(N \geq 2)$, early results suggested that quantum fluctuations always destroy long-range order at $p_{c}$ [9, 10, 11]. However, Sandvik [12] showed that the percolating cluster in a $2 \mathrm{~d}$ diluted Heisenberg quantum antiferromagnet (QAFM) is ordered at $p_{c}$ (see also Ref. 13]). If quantum fluctuations are enhanced, e.g., by an interlayer coupling in a bilayer QAFM 14, 15], a multicritical point at $\left(p_{c}, g^{*}\right)$ arises, similar to the transverse field Ising case. The behavior at the generic quantum phase transition occurring for $g>g^{*}, p<p_{c}$ (solid line in Fig. 11) and at the multi-critical point at $\left(p_{c}, g^{*}\right)$ has been investigated in the past [14, 15, 16]. In contrast, the percolation quantum phase transition at $p_{c}$ and $g<g^{*}$ which can be observed, e.g., in diluted 2d QAFMs such as $\mathrm{La}_{2} \mathrm{Cu}_{1-p}(\mathrm{Zn}, \mathrm{Mg})_{p} \mathrm{O}_{4}$ [17] has received less attention.

In this Letter, we show that the interplay of geometric criticality and quantum fluctuations leads to a novel universality class for this percolation quantum phase transition. Even though the transition is driven entirely by geometric criticality of the underlying classical percolation problem, quantum fluctuations enhance the singularities in all quantities involving dynamic correlations. As a result, the susceptibility exponent $\gamma$ as well as $\alpha$, $\delta$ and $\eta$ differ from the classical percolation values, and the dynamical exponent $z$ is nontrivial. In contrast, the order parameter and correlation lengths exponents are classical, $\beta=\beta_{c}$ and $\nu=\nu_{c}$ 18. In the remainder of this Letter we sketch the derivation of these results via a scaling approach (we have also performed detailed explicit calculations) and calculate critical exponent values in $2 \mathrm{~d}$ and $3 \mathrm{~d}$. In addition, we make predictions for various experimentally relevant observables.

Our starting point is a general $O(N)$ quantum rotor model $(N \geq 2)$ on a $d$-dimensional hypercubic lattice with nearest neighbor interactions as arises as low-energy theory of a Heisenberg quantum antiferromagnet [19]. 


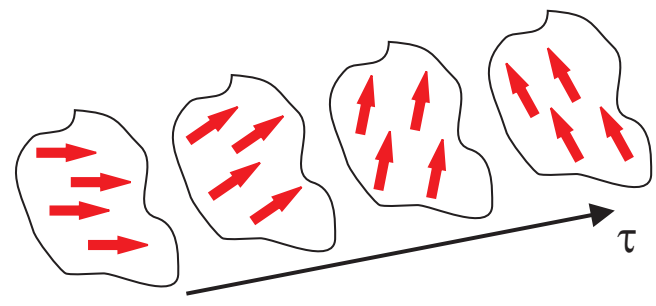

FIG. 2: For $g<g^{*}$, a percolation cluster acts as a compact object. The spins are correlated in space but collectively fluctuate in imaginary time $\tau$.

The action reads

$$
\begin{aligned}
\mathcal{A} & =\int d \tau \sum_{\langle i j\rangle} J \epsilon_{i} \epsilon_{j} \mathbf{S}_{i}(\tau) \cdot \mathbf{S}_{j}(\tau)+\sum_{i} \epsilon_{i} \mathcal{A}_{\mathrm{dyn}}\left[\mathbf{S}_{i}\right] \\
\mathcal{A}_{\mathrm{dyn}}[\mathbf{S}] & =\frac{T}{g} \sum_{n=-\infty}^{\infty}\left|\omega_{n}\right|^{2 / z_{0}} \mathbf{S}\left(\omega_{n}\right) \mathbf{S}\left(-\omega_{n}\right)
\end{aligned}
$$

Here $\mathbf{S}_{i}(\tau)$ is a $N$-component unit vector at lattice site $i$ and imaginary time $\tau$. The quenched random variable $\epsilon_{i}$ describes the site dilution. It takes the values 0 and 1 with probabilities $p$ and $(1-p)$, respectively. The parameter $g$ in the dynamic term measures the strength of the quantum fluctuations [20], $\omega_{n}$ are bosonic Matsubara frequencies, and $z_{0}$ is the dynamical exponent of the clean system. We are mostly interested in the case $z_{0}=1$ (undamped rotor dynamics) but our results also apply for $0<z_{0}<2$ where $z_{0} \rightarrow 0$ corresponds to the classical limit while $z_{0}=2$ is the case of overdamped spin dynamics (occurring in itinerant magnets 21, 22]).

Let us start our discussion of the percolation quantum phase transition at $p=p_{c}$ and $g<g^{*}$ by considering order parameter $m$ and correlation length $\xi$. Long range magnetic order can only develop on the infinite percolation cluster; all finite clusters are completely decoupled. Since for $g<g^{*}$ the infinite cluster remains magnetically ordered for all $p \leq p_{c}$, the total order parameter is proportional to the number of sites in the infinite cluster, $m \sim P_{\infty}(p) \sim\left(p_{c}-p\right)^{\beta_{c}}$. Thus, $\beta=\beta_{c}$. To determine the magnetic correlation length $\xi$ we note that the correlations cannot extend beyond the connectedness length $\xi_{c}$ of the percolating lattice because the rotors on different clusters are decoupled. On the other hand, for $g<g^{*}$, all rotors on the same cluster are strongly correlated in space even though they collectively fluctuate in time (see Fig. (2). We thus conclude $\xi \sim \xi_{c}$ and $\nu=\nu_{c}$.

To study quantities involving dynamic correlations, we develop a scaling theory of the free energy, contrasting classical and quantum cases. Consider a single percolation cluster of finite size (number of sites) $s$. In a classical magnet for $T \rightarrow 0$, the free energy of such a cluster has the scaling form $F_{s}^{\mathrm{c}}(h, T)=T \Phi(h s / T)$, where $h$ is the field conjugate to the order parameter and $\Phi(x)$ a dimensionless function. The susceptibility $\chi_{s}^{\mathrm{c}}$ follows from taking the second derivative with respect to $h$; this gives $\chi_{s}^{\mathrm{c}} \sim s^{2} / T$ as expected. Let us now consider the quantum case. For $g<g^{*}$, a quantum rotor cluster of size $s$ can be treated as a compact object that fluctuates in time only (see Fig. 2) and is described by a $0+1$ dimensional nonlinear sigma model (NLSM) with the action 23, 24]

$$
\mathcal{A}_{s}=s \mathcal{A}_{\mathrm{dyn}}[\mathbf{S}]+h s \int d \tau S^{(1)}(\tau) .
$$

Here, $S^{(1)}$ is one component of $\mathbf{S}$. Dimensional analysis shows that the free energy of a cluster of size $s$ is

$$
F_{s}(g, h, T)=g^{\varphi} s^{-\varphi} \Phi\left(h s^{1+\varphi} g^{-\varphi}, T s^{\varphi} g^{-\varphi}\right)
$$

with exponent $\varphi=z_{0} /\left(2-z_{0}\right)$ (for $\left.z_{0}<2\right)$. This result can be obtained by rescaling imaginary time, $\tau \rightarrow$ $g^{\varphi} s^{-\varphi} \tau$, which eliminates the factor $(s / g)$ in the dynamic part of the action, and leads to a dimensionless field strength $\left(h s^{1+\varphi} / g^{\varphi}\right)$ and temperature $\left(T s^{\varphi} / g^{\varphi}\right)$. The resulting order parameter susceptibility $\chi_{s} \sim s^{2+\varphi}$ of a quantum spin cluster diverges more strongly with $s$ than that of a classical spin cluster. We have obtained the same results from an explicit large- $N$ calculation [25] and from a renormalization group analysis of the NLSM (2) at its strong coupling fixed point [24, 26]

After having analyzed a single cluster we turn to the full percolation problem. The total free energy can be obtained by summing the cluster free energy $F_{s}$ over all percolation clusters. From classical percolation theory, we know that close to $p_{c}$ the number $n_{s}$ of occupied clusters of size $s$ per lattice site obeys the scaling form

$$
n_{s}(t)=s^{-\tau_{c}} f\left(t s^{\sigma_{c}}\right) .
$$

Here $t=p-p_{c}$, and $\tau_{c}$ and $\sigma_{c}$ are classical percolation exponents. The scaling function $f(x)$ is constant for small $x$ and drops off rapidly for large $x$. All classical percolation exponents are determined by $\tau_{c}$ and $\sigma_{c}$ including $\nu_{c}=\left(\tau_{c}-1\right) /\left(d \sigma_{c}\right)$ and the fractal dimension $D_{f}=d /\left(\tau_{c}-1\right)$ of the percolating cluster [1].

We first briefly recapitulate the percolation transition of a classical magnet at $T=0$. The total free energy is given by the sum $F^{\mathrm{c}}(t, h, T)=\sum_{s} n_{s}(t) F_{s}^{\mathrm{c}}(h, T)$. Rescaling $s \rightarrow s / b^{D_{f}}$ and taking the limit $T \rightarrow 0$ yields

$$
F^{\mathrm{c}}(t, h)=b^{-d} F^{\mathrm{c}}\left(t b^{1 / \nu_{c}}, h b^{D_{f}}\right) .
$$

The critical behavior of thermodynamic quantities can be obtained by taking the appropriate derivatives. All critical exponents coincide with the classical (geometric) percolation exponents which is expected because in a classical magnet at $T=0$ geometric fluctuations are the only fluctuations present [1, 3,, 5 .

We next generalize these ideas to the percolation quantum phase transition in the quantum rotor model (11) 
for $g<g^{*}$. Averaging the free energy (3) of a quantum rotor cluster over all cluster sizes, $F(t, h, T)=$ $\sum_{s} n_{s}(t) F_{s}(g, h, T)$, and rescaling $s \rightarrow s / b^{D_{f}}$ yields

$$
F(t, h, T)=b^{-(d+z)} F\left(t b^{1 / \nu}, h b^{\left(D_{f}+z\right)}, T b^{z}\right)
$$

with the correlation length exponent identical to the classical value, $\nu=\nu_{c} . z=\varphi D_{f}$ plays the role of the dynamic critical exponent. We have verified this interpretation by calculating the correlation time $\xi_{\tau}$ and showing $\xi_{\tau} \sim \xi^{z}$. Comparing the scaling form of the free energy (6) with its classical counterpart (5) we notice that the quantum theory is obtained from the classical one, as usual, by replacing $d$ by $d+z$. In addition, one should replace $D_{f}$ by $D_{f}+z$. This occurs since the magnetic field couples to the spins at all "imaginary time points".

The critical exponents at the percolation quantum phase transition can be obtained by taking the appropriate derivatives of the free energy. All exponents are fully determined by two classical percolation exponents (e.g., the fractal dimension $D_{f}$ and the correlation length exponent $\nu=\nu_{c}$ ) as well as the dynamic exponent $z$ (the classical exponents are recovered by setting $z=0)$ :

$$
\begin{aligned}
2-\alpha & =(d+z) \nu \\
\beta & =\left(d-D_{f}\right) \nu \\
\gamma & =\left(2 D_{f}-d+z\right) \nu \\
\delta & =\left(D_{f}+z\right) /\left(d-D_{f}\right) \\
2-\eta & =2 D_{f}-d+z .
\end{aligned}
$$

The exponents $\alpha, \gamma, \delta$, and $\eta$ are modified compared to classical percolation, while $\beta$ depends only on $d-D_{f}$ and is thus unchanged. The exponents fulfill the usual scaling laws, and hyperscaling is valid for $d<6$. This means, the upper critical dimension is identical to its classical value, it is not changed by the substitution $d \rightarrow d+z$.

The percolation exponents are known exactly in $2 \mathrm{~d}[\mathbf{1}]$, as is the dynamical exponent $z=D_{f} \varphi=D_{f} z_{0} /\left(2-z_{0}\right)$. Thus, all exponents for the quantum phase transition can be determined exactly. For higher dimensions, $\nu$ and $D_{f}$ are known numerically with good accuracy [1]. Table I shows exponent values for $z_{0}=1 \mathrm{in} 2 \mathrm{~d}$ and $3 \mathrm{~d}$. The dependence of the critical exponents on $z_{0}$ is shown in Fig. 3. In the limit $z_{0} \rightarrow 0$, the quantum exponents become identical to the classical percolation exponents, and the dynamical exponent $z$ vanishes. For $z_{0} \rightarrow 2$, the dynamical exponent $z$ as well as $\gamma$ and $\delta$ diverge, indicating activated scaling at an infinite-randomness critical point as in the Ising case [8].

The scaling form (6) of the free energy also determines the temperature dependence of the order parameter susceptibility $\chi(t, T)=T^{-\gamma /(\nu z)} \Theta_{\chi}\left(t^{\nu z} / T\right)$ and the heat capacity $C=T\left(\partial^{2} F / \partial T^{2}\right)=T^{d / z} \Theta_{c}\left(t^{\nu z} / T\right)$ with scaling functions $\Theta_{\chi, c}(x)$. At $p=p_{c}$ (or for $\left.p-p_{c}<T^{1 /(\nu z)}\right)$, this yields $\chi(T) \propto T^{-\gamma /(\nu z)}$ and $C(T) \propto T^{d / z}$. Spin

\begin{tabular}{c|cc|cc}
\hline & \multicolumn{2}{|c}{$2 \mathrm{~d}$} & \multicolumn{2}{c}{$3 \mathrm{~d}$} \\
Exponent & classical & quantum & classical & quantum \\
\hline$\alpha$ & $-2 / 3$ & $-115 / 36$ & -0.62 & -2.83 \\
$\beta$ & $5 / 36$ & $5 / 36$ & 0.417 & 0.417 \\
$\gamma$ & $43 / 18$ & $59 / 12$ & 1.79 & 4.02 \\
$\delta$ & $91 / 5$ & $182 / 5$ & 5.38 & 10.76 \\
$\nu$ & $4 / 3$ & $4 / 3$ & 0.875 & 0.875 \\
$\eta$ & $5 / 24$ & $-27 / 16$ & -0.06 & -2.59 \\
$z$ & - & $91 / 48$ & - & 2.53 \\
\hline
\end{tabular}

TABLE I: Critical exponents at the classical and quantum percolation transition for $d=2$ and 3 in case of $z_{0}=1$.

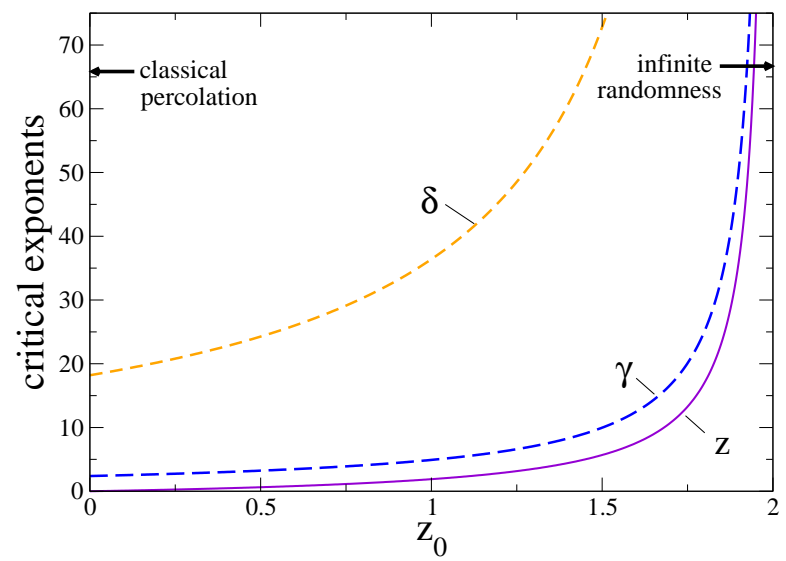

FIG. 3: Exponents $\gamma, \delta$, and $z$ as functions of $z_{0}$ in 2 d. The limiting cases $z_{0} \rightarrow 0$ and $z_{0} \rightarrow 2$ are explained in the text.

wave type excitations within a cluster have typical energies $\omega_{\mathrm{sw}} \propto s^{-z_{0} / D_{f}}$ that are large compared to the energy gap $\omega_{0} \propto s^{-\varphi}=s^{-z / D_{f}}$ of the cluster because $z>z_{0}$ (for $\left.z_{0}=1\right)$. Thus, spin waves that were shown to be important away from $p_{c}$ 27], are subleading at $p_{c}$ as can also directly be seen from the heat capacity contributions.

A scaling theory for the dynamic susceptibility can be derived following similar arguments. We obtain $\chi(t, \omega, T)=b^{\gamma / \nu} \chi\left(t b^{1 / \nu}, \omega b^{z}, T b^{z}\right)$ which at $p_{c}$ yields on the real frequency axis:

$$
\operatorname{Im} \chi\left(\omega+i 0^{+}\right)=\omega^{-\gamma /(\nu z)} \Omega(\omega / T) .
$$

with dimensionless scaling function $\Omega(x)$. Eq. (12) implies that low-temperature inelastic neutron scattering experiments at the location of the Bragg peak should see a sharp increase $\sim \omega^{-\gamma /(\nu z)}$ in the scattering intensity for $\omega \rightarrow 0$, while $\omega^{\gamma /(\nu z)} \operatorname{Im} \chi\left(\omega+i 0^{+}\right)$obeys $\omega / T$-scaling.

In the remaining paragraphs we discuss experiments, simulations and the generality of our results. The NLSM (11) is the exact low-energy theory of a dimer-diluted bilayer Heisenberg QAFM [14, 15, 16], or in $d>2$, that of an appropriately bond-diluted system. One candidate material in $3 \mathrm{~d}$ is $(\mathrm{Tl}, \mathrm{K}) \mathrm{CuCl}_{3}$ 28]; interesting quasi-2d compounds are $\mathrm{SrCu}_{2}\left(\mathrm{BO}_{3}\right)_{2}$ or $\mathrm{BaCuSi}_{2} \mathrm{O}_{6}$, where suitable dopants remain to be found. In addition, the ac- 
tion (11) describes Josephson junction arrays with random missing junctions and diluted bosons in optical lattices.

If magnetic ions are replaced by nonmagnetic ones in a QAFM, e.g., $\mathrm{Cu}$ by $\mathrm{Zn}$ or $\mathrm{Mg}$ in $\mathrm{La}_{2} \mathrm{CuO}_{4}$ or $\mathrm{YBa}_{2} \mathrm{Cu}_{3} \mathrm{O}_{6}$, impurity induced moments arise which leads to random Berry phases in the NLSM 29]. These moments produce Curie type contributions that dominate the low-temperature behavior in the disordered phase $p>p_{c}$. However, at $p=p_{c}$, their contributions are subleading compared to the critical singularities. Thus, our theory also describes the percolation quantum phase transition in site-diluted systems, and we propose to study QAFMs such as $\mathrm{La}_{2} \mathrm{Cu}_{1-p}(\mathrm{Zn}, \mathrm{Mg})_{p} \mathrm{O}_{4}$. Recent measurements [17] indeed gave a nontrivial dynamical exponent (with a somewhat smaller value of $z \approx 1.4$ ). The measurement of several observables may help to identify the scaling regime and possibly refine the value of $z$. An issue that may limit the applicability of our theory very close to $p_{c}$ is dilution induced frustration.

We now turn to simulations, focusing on the dynamical exponent z. Early series expansions [9] gave values in the range of 1.5 to 2 . Kato et al. [11] obtained spindependent values between 1.3 and 2.5 but under the assumption that the critical percolation cluster is not longrange ordered. Recent simulations of a bilayer QAFM [14] and of a bond-diluted 2d QAFM 30] gave values of about 1.9, in excellent agreement with our theory. These two papers also gave arguments for $z=D_{f}$ as obtained in our theory for $z_{0}=1$.

Finally, we discuss the generality of our theory. To derive the nonclassical scaling theory (6) of the percolation quantum phase transition we only relied on the fact that the energy gap $\omega_{0}$ of a cluster depends on its size $s$ via a power law, $\omega_{0} \propto s^{-\varphi}$ with $\varphi>0$. If $\varphi$ diverges, which happens in our model for $z_{0}=2$, the relation between the gap and $s$ becomes exponential. The transition is then of infinite-randomness type as in the diluted transverse field Ising model 8]. These observations agree with a general classification of dirty phase transitions according to the effective dimensionality of the droplets or clusters 25]. For $z_{0}<2$, the clusters (which are finite in space but infinite in imaginary time) are below the lower critical dimension $d_{c}^{-}$of the problem resulting in powerlaw scaling (and exponentially weak Griffiths effects). In contrast, for $z_{0}=2$, the clusters are exactly at $d_{c}^{-}$. This leads to activated scaling and power-law Griffiths effects.

We acknowledge helpful discussions with M. Greven, M.L. Lyra, A. Sandvik, J. Toner, and M. Vojta. Parts of this work have been performed at the Aspen Center for Physics and the Kavli Institute for Theoretical Physics, Santa Barbara. This work was supported in part by Ames Laboratory, operated for the U.S. Department of Energy by Iowa State University under Contract No. W-7405-Eng-82 (J.S.), by the NSF under grant Nos. DMR-0339147 (T.V.) and PHY99-07949 (KITP SB) as well as by Research Corporation.
[1] D. Stauffer and A. Aharony, Introduction to Percolation Theory (Taylor \& Francis, London, 1994).

[2] Order at $T>0$ requires $d>2$ (continuous symmetry) or $d>1$ (Ising symmetry).

[3] T.K. Bergstresser, J. Phys. C 10, 3831 (1977).

[4] M.J. Stephen and G.S. Grest, Phys. Rev. Lett. 38, 567 (1977).

[5] Y. Gefen, B.B. Mandelbrot, and A. Aharony, Phys. Rev. Lett. 45, 855 (1980).

[6] For Ising symmetry, this appears counterintuitive as the dimension of the critical percolation cluster is larger than 1. However, for fractals, topological factors like ramification are important for magnetic ordering [5].

[7] A.B. Harris, J. Phys. C 7, 3082 (1974); R.B. Stinchcombe, J. Phys. C 14, L263 (1981); R.R. dos Santos, J. Phys. C 15, 3141 (1982).

[8] T. Senthil and S. Sachdev, Phys. Rev. Lett. 77, 5292 (1996).

[9] C.C. Wan, A.B. Harris, and J. Adler, J. Appl. Phys. 69, 5191 (1991).

[10] C. Yasuda and A. Oguchi, J. Phys. Soc. Jpn. 68, 2773 (1999); Y.C. Chen and A.H. Castro Neto, Phys. Rev. B 61, R3772 (2000).

[11] K. Kato et al., Phys. Rev. Lett. 84, 4204 (2000).

[12] A.W. Sandvik, Phys. Rev. Lett. 86, 3209 (2001); Phys. Rev. B 66, 024418 (2002).

[13] N. Bray-Ali et al., cond-mat/0507587.

[14] A.W. Sandvik, Phys. Rev. Lett. 89, 177201 (2002).

[15] O.P. Vajk and M. Greven, Phys. Rev. Lett. 89, 177202 (2002).

[16] R. Sknepnek, T. Vojta, and M. Vojta, Phys. Rev. Lett. 93, 097201 (2004).

[17] O.P. Vajk et al., Science 295, 1691 (2002).

[18] In the following, classical percolation exponents carry a subscript $c$ while the magnetic exponents at our percolation quantum phase transition do not carry a subscript.

[19] S. Sachdev, Quantum phase transitions (Cambridge University Press, Cambridge, 1999), chapter 13, pp. 240.

[20] For simple quantum antiferromagnets, $g$ can be related to the spin value $S$. In the large- $S$ limit, $g \sim 1 / S^{2}[19]$.

[21] J. Hertz, Phys. Rev. B 14, 1165 (1976).

[22] In a metal, a percolation scenario may be hard to realize because of the long-range RKKY interaction that can change the critical behavior, see V. Dobrosavljevic and E. Miranda, Phys. Rev. Lett. 94, 187203 (2005).

[23] D. S. Fisher, Phys. Rev. B 39, 11783 (1989).

[24] J. Toner, private communication.

[25] T. Vojta and J. Schmalian, Phys. Rev. B 72, 045438 (2005).

[26] This analysis gives the leading behavior exactly as the stable fixed point does not have anomalous dimensions.

[27] A. L. Chernyshev, Y. C. Chen, A. H. Castro Neto, Phys. Rev B 65, 104407 (2002).

[28] A. Oosawa and H. Tanaka, Phys. Rev. B 65, 184437 (2002).

[29] S. Sachdev and M. Vojta, in Proc. of the XIII International Congress on Mathematical Physics, edited by A. Fokas et al. (International Press, Boston, 2001).

[30] R. Yu, T. Roscilde, and S. Haas, Phys. Rev. Lett. 94, 197204 (2005). 